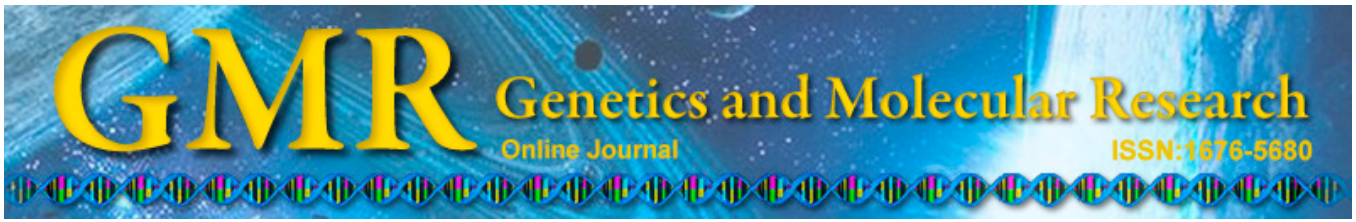

\title{
Association between the XRCC1 Arg399GIn polymorphism and risk of cervical carcinoma: a meta-analysis
}

\author{
D.Y. Liu*, H.C. Liang* and X.M. Xiao \\ Department of Gynecology and Obstetrics, \\ The First Affiliated Hospital of Jinan University, Guangzhou, \\ Guangdong, China \\ *These authors contributed equally to this study. \\ Corresponding author: X.M. Xiao \\ E-mail: xiaomed@126.com
}

Genet. Mol. Res. 14 (3): 9821-9828 (2015)

Received December 12, 2014

Accepted May 20, 2015

Published August 19, 2015

DOI http://dx.doi.org/10.4238/2015.August.19.15

\begin{abstract}
Numerous studies have evaluated the association between Arg399Gln polymorphism of DNA repair gene XRCC1 and cervical carcinoma risk. However, the specific association is still controversial. To assess the relationship between XRCC1 Arg399Gln polymorphism and cervical carcinoma, we conducted a comprehensive meta-analysis of 10 case-control studies with 2051 cervical carcinoma cases and 2919 controls. Meta-analysis results based on all the studies showed a significant association between XRCC1 Arg399Gln polymorphism and cervical carcinoma risk (GlnGln $v$ s ArgArg: OR $=1.29,95 \% \mathrm{CI}$ $=0.90-1.85 ;$ GlnGln vs ArgGln: $\mathrm{OR}=1.15,95 \% \mathrm{CI}=0.93-1.43$; the dominant model: $\mathrm{OR}=0.80,95 \% \mathrm{CI}=0.66-0.99$; the recessive model: $\mathrm{OR}=1.18,95 \% \mathrm{CI}=0.93-1.49)$. In the subgroup analysis by ethnicity, the results also showed significant association between XRCC1 Arg399Gln polymorphism and susceptibility to cervical carcinoma in both Caucasian and Asian populations. The Arg399Gln polymorphism in the XRCC1 gene may be related to the increased risk of cervical
\end{abstract}


carcinoma. Conclusive evidence on the effects of the variants in cervical carcinoma should be addressed in further studies.

Key words: XRCC1; Gene polymorphism; Cervical carcinoma; Meta-analysis

\section{INTRODUCTION}

Cervical carcinoma is one of the most common gynecological malignancies, and its prevalence has increased during the last 10 years (Boyle et al., 2003). More than $85 \%$ of the global burden occurs in developing countries, where it accounts for $13 \%$ of all female cancers (Suh et al., 2010). Despite much investigation, the causes are not yet fully understood. It is well established that human papilloma virus (HPV) infection is a necessary event for the development of cervical cancer (Crosbie et al., 2013). However, only a small percentage of infected women will eventually develop cervical cancer. Infection with HPV alone is not sufficient for the development of cervical carcinoma. Evidence is accumulating that genetic variants in genes controlling carcinogen metabolism, DNA repair and cell proliferation or apoptosis may be important in determining individual susceptibility to the occurrence and progression of cervical carcinoma (Zucchetto et al., 2009; Yang et al., 2010).

X-ray cross-complementing gene 1 (XRCC1), located on chromosome 19 (19q13.2), is involved in the repair of DNA base damage and single-strand DNA breaks by binding DNA ligase III at its carboxyl end and DNA polymerase $b$ and poly (ADP-ribose) polymerase at the site of damaged DNA (Caldecott et al., 1996). In addition, XRCC1 is known to participate in base excision repair of small lesions such as oxidized or reduced bases, fragmented or nonbulky adducts, and lesions caused by methylating agents (Caldecott, 2003). Although more than 300 validated SNPs have been identified and described in the XRCC1 gene, only three common SNPs have been extensively studied: Arg194Trp (rs 1799782, C/T substitution at position 26304 in exon 6), Arg280His (rs25489, G/A substitution at position 27466 in exon 9) and Arg399Gln (rs25487, G/A substitution at position 28152 in exon 10). These polymorphisms could alter XRCC1 function and result in altered efficiency of the protein, eventually inducing the development of cancer.

Over the past decade, a number of epidemiological studies demonstrated that XRCC1 Arg399Gln polymorphism was associated with susceptibility to gastric, lung, breast cancer, and other types of cancers (Park et al., 2002; Kim et al., 2002; Lee et al., 2012). However, little is known regarding the association between Arg399Gln polymorphism of XRCC1 gene and susceptibility to cervical carcinoma. To help clarify the inconsistent findings, with the publication of several recent case-control studies, we conducted this meta-analysis of XRCC1 Arg399Gln polymorphism and the risk of cervical carcinoma.

\section{MATERIAL AND METHODS}

\section{Selection of studies}

We searched the PubMed, Embase and China National Knowledge Infrastructure databases for all articles on the association between XRCC1 Arg399Gln polymorphism and cervical carcinoma risk (last search update November 2014). The following key words were used: 
"cervical carcinoma" or "cervical cancer", "XRCC1" or "Arg399Gln" and "polymorphism" or "variant". Publication date and publication language were not restricted in our search. If more than one article was published by the same author using the same case series, we selected the study where the most individuals were investigated.

\section{Inclusion and exclusion criteria}

Any patient-associated study, regardless of sample size, was included if it met the following criteria: i) case-control studies were conducted to evaluate the association between the XRCC1 Arg399Gln polymorphism and the risk of cervical carcinoma; ii) sufficient genotype data were presented to calculate the odds ratios (ORs) and 95\% confidence intervals (CIs); and iii) the paper clearly described the sources of cases and controls. Major reasons for the exclusion of studies were i) no control, ii) duplicated studies, and iii) sufficient data were not reported.

\section{Data extraction}

All data were extracted independently by 2 reviewers according to the inclusion criteria listed above. Disagreements were resolved by discussion between the 2 reviewers. The following characteristics were collected from each study: first author, year of publication, country of sample, ethnicity, number of cases and controls, number of genotypes and evidence of Hardy-Weinberg equilibrium (HWE), which are given in Table 1. If original genotype frequency data were unavailable in relevant articles, an email was sent to the corresponding author for additional data.

\section{Statistical analysis}

Statistical analysis was conducted using STATA 12.0 (Stata Corp. LP, College Station, TX, USA); $\mathrm{P}<0.05$ was considered to be statistically significant. HWE in the controls was tested by the chi-square test for goodness of fit, and $\mathrm{P}<0.05$ indicated significant disequilibrium. The strength of the associations between XRCC1 Arg399Gln polymorphism and susceptibility to cervical carcinoma was estimated by OR and 95\%CI under a homozygote comparison (GlnGln vs ArgArg), a heterozygote comparison (GlnGln vs ArgGln), a dominant model (ArgArg + ArgGln vs GlnGln), and a recessive mode (GlnGln + ArgGln vs ArgArg) between groups. Heterogeneity among studies was assessed by the $I^{2}$ statistic, which described the proportion of total variation attributable to between-study differences or heterogeneity as opposed to random error or chance. If $I^{2}>50 \%$, the DerSimonian and Laird random-effect model was adopted as the pooling method; otherwise, the fixed-effect model was used. Sensitivity analysis was performed through random-effect model values compared to the fixed effect. Publication bias was examined using Begg's funnel plot $(\mathrm{P}<0.05$ was considered representative of statistically significant publication bias).

\section{RESULTS}

\section{Study characteristics}

Through screening the title and reading the abstract and the entire article, 10 eligible 
articles were selected for this meta-analysis (Wu et al., 2004; Niwa et al., 2005; Huang et al., 2007; Jiang et al., 2009; Xiao et al., 2010; Wannapa et al., 2011; Ma et al., 2011; Barbisan et al., 2011; Roszak et al., 2011; Zhang et al., 2012). Study characteristics are summarized in Figure 1. All studies included were case-control studies that evaluated the association between XRCC1 Arg399Gln polymorphism and susceptibility to cervical carcinoma. The published year of the studies included ranged from 2000 to 2014. The source of controls was based on healthy populations. There were two studies of Europeans (Barbisan et al., 2011; Roszak et al., 2011) and eight of Asians (Wu et al., 2004; Niwa et al., 2005; Huang et al., 2007; Jiang et al., 2009; Xiao et al., 2010; Wannapa et al., 2011; Ma et al., 2011; Zhang et al., 2012). The genotype distributions in the controls of all studies were in agreement with HWE. The information from these 10 studies and the numbers of cases and controls with ArgArg, ArgGln and GlnGln genotypes reported in each study are all presented in Table 1.

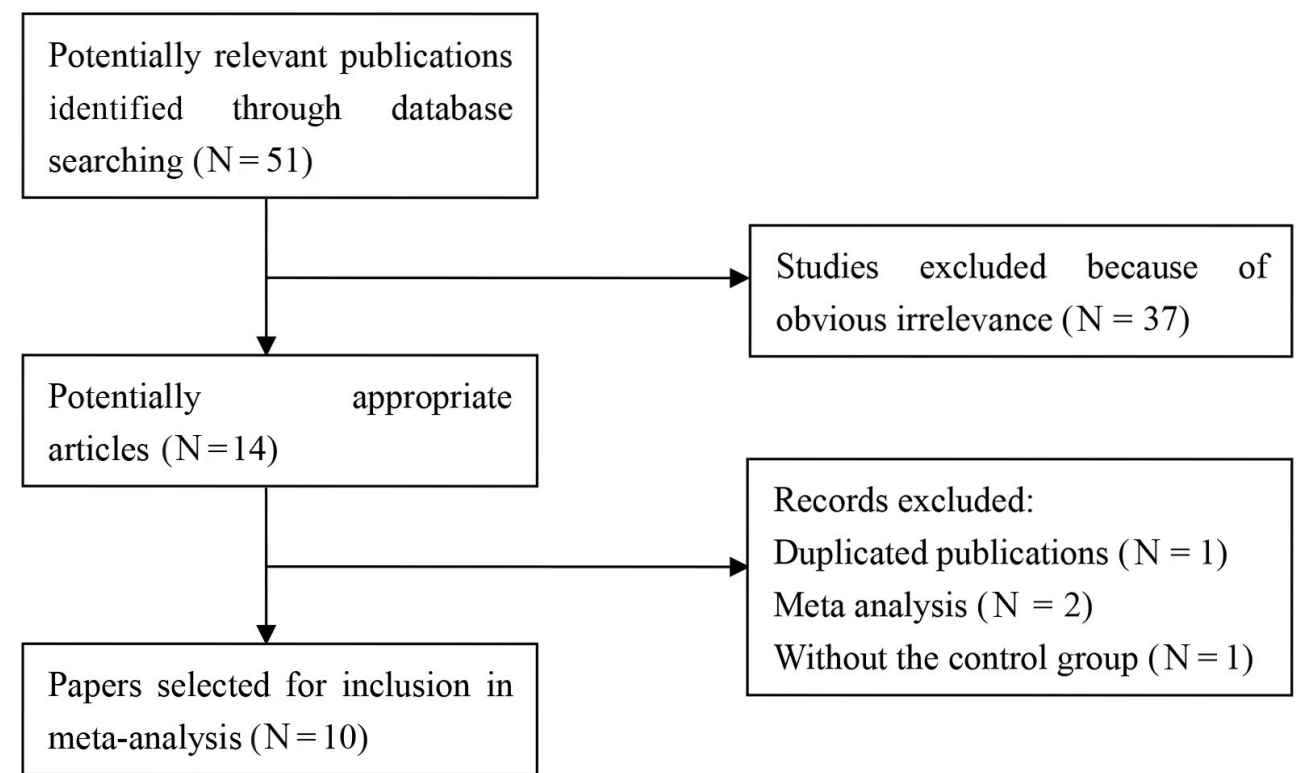

Figure 1. Flow diagram of study searching and selection process.

Table 1. Characteristics of the studies included for meta-analysis.

\begin{tabular}{|c|c|c|c|c|c|c|c|c|c|c|c|}
\hline \multirow[t]{2}{*}{ Study included } & \multirow[t]{2}{*}{ Year } & \multirow[t]{2}{*}{ Area } & \multirow[t]{2}{*}{ Race } & \multirow[t]{2}{*}{ Case/Control } & \multicolumn{3}{|c|}{ Genotypes for cases } & \multicolumn{3}{|c|}{ Genotypes for controls } & \multirow[t]{2}{*}{ HWE test } \\
\hline & & & & & ArgArg & ArgGln & GlnGln & ArgArg & ArgGln & GlnGln & \\
\hline Wu et al. & 2004 & China & Asian & $100 / 196$ & 54 & 38 & 8 & 114 & 73 & 9 & 0.53 \\
\hline Niwa et al. & 2005 & Japan & Asian & $131 / 320$ & 69 & 49 & 13 & 185 & 109 & 26 & 0.09 \\
\hline Huang et al. & 2007 & China & Asian & $539 / 800$ & 289 & 203 & 47 & 528 & 235 & 37 & 0.10 \\
\hline Jiang et al. & 2009 & China & Asian & $436 / 503$ & 228 & 184 & 24 & 268 & 194 & 41 & 0.48 \\
\hline Xiao et al. & 2010 & China & Asian & $162 / 183$ & 91 & 56 & 15 & 94 & 68 & 21 & 0.12 \\
\hline Wannapa et al. & 2011 & Thailand & Asian & $111 / 118$ & 66 & 41 & 4 & 69 & 44 & 5 & 0.54 \\
\hline Ma et al. & 2011 & China & Asian & $200 / 200$ & 108 & 76 & 16 & 133 & 55 & 12 & 0.06 \\
\hline Barbisan et al. & 2011 & Argentina & Caucasian & $103 / 114$ & 54 & 31 & 18 & 37 & 59 & 18 & 0.49 \\
\hline Roszak et al. & 2011 & Poland & Caucasian & $189 / 308$ & 49 & 101 & 39 & 116 & 152 & 40 & 0.37 \\
\hline Zhang et al. & 2012 & China & Asian & $80 / 177$ & 43 & 31 & 6 & 109 & 58 & 10 & 0.54 \\
\hline
\end{tabular}




\section{Meta analysis results}

The results on the associations between Arg399Gln polymorphism of DNA repair gene XRCC1 and cervical carcinoma risk, the heterogeneity test and the test of publication bias are shown in Figure 2 and Table 2. The combined results based on all studies showed that XRCC1 Arg399Gln polymorphism is associated with increased cervical carcinoma risk in different genetic models (GlnGln vs ArgArg: $\mathrm{OR}=1.29,95 \% \mathrm{CI}=0.90-1.85$; GlnGln $v s$ ArgGln: $\mathrm{OR}=1.15,95 \% \mathrm{CI}=0.93-1.43$; the dominant model: $\mathrm{OR}=0.80,95 \% \mathrm{CI}=0.66-0.99$; the recessive model: $\mathrm{OR}=1.18,95 \% \mathrm{CI}=0.93-1.49)$. Sensitivity analyses were conducted by altering the statistical models. No material alteration was detected, indicating that our results were statistically robust.

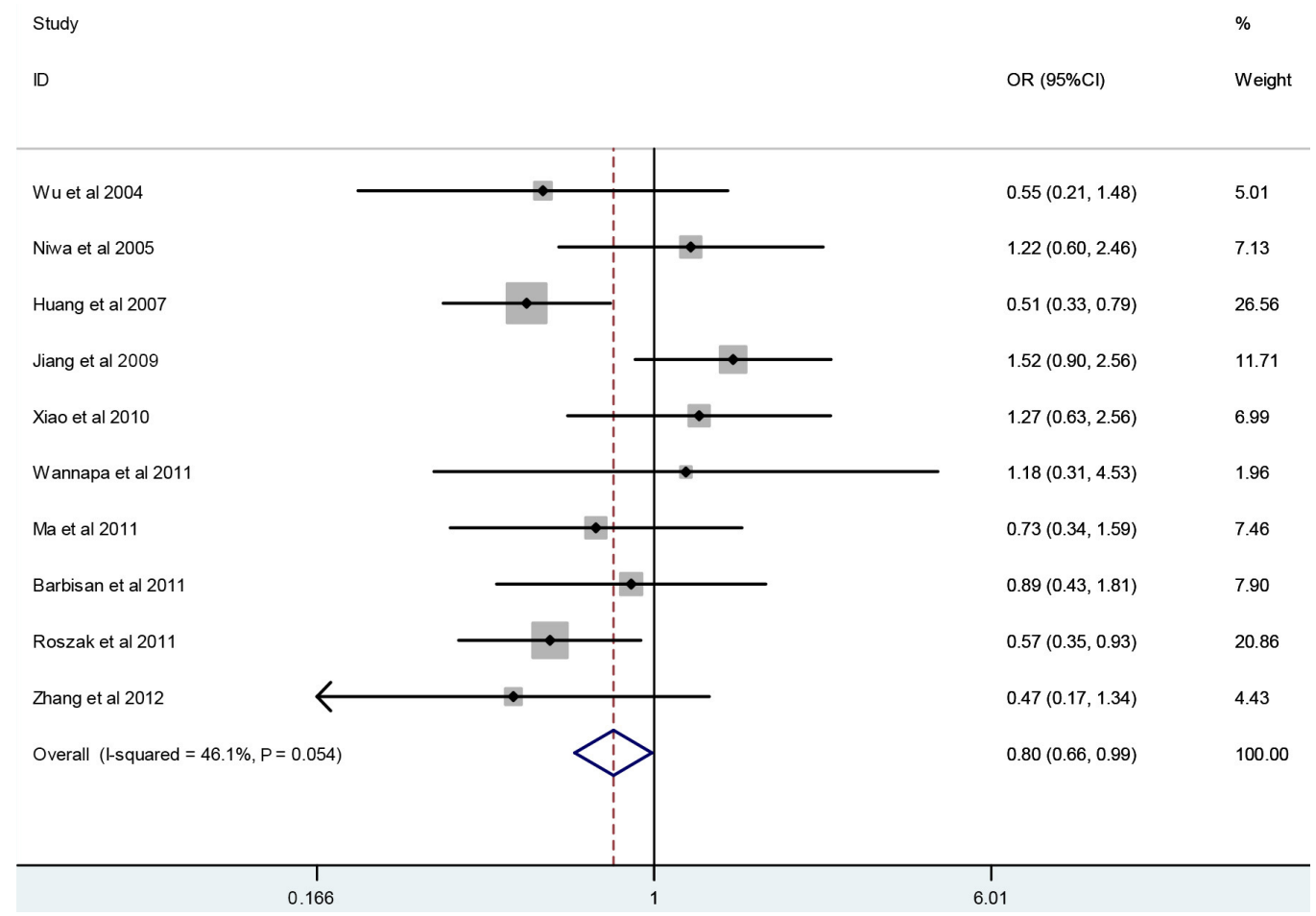

Figure 2. Forest plot of cervical carcinoma risk associated with XRCC1 Arg399Gln polymorphism in the dominant model.

In the subgroup analysis based on ethnicity, results of subgroup analysis confirmed that there were significant associations between XRCC1 Arg399Gln polymorphism and cervical carcinoma risk in both Asian populations (GlnGln $v s$ ArgArg: $\mathrm{OR}=1.03,95 \% \mathrm{CI}=$ 0.80-1.33; GlnGln vs ArgGln: $\mathrm{OR}=1.03,95 \% \mathrm{CI}=0.78-1.35$; the dominant model: $\mathrm{OR}=$ $0.87,95 \% \mathrm{CI}=0.59-1.26$; the recessive model: $\mathrm{OR}=1.25,95 \% \mathrm{CI}=1.02-1.53)$ and Caucasian populations (GlnGln vs ArgArg: $\mathrm{OR}=1.30,95 \% \mathrm{CI}=0.40-4.26$; GlnGln vs ArgGln: $\mathrm{OR}=$ $1.58,95 \% \mathrm{CI}=1.03-2.43$; the dominant model: $\mathrm{OR}=0.66,95 \% \mathrm{CI}=0.44-0.99$; the recessive model: $\mathrm{OR}=0.88,95 \% \mathrm{CI}=0.22-3.39)$. 
Table 2. Summary ORs and $95 \% \mathrm{CI}$ of the XRCC1 Arg399Gln polymorphism and cervical carcinoma risk.

\begin{tabular}{|c|c|c|c|c|c|c|c|c|c|}
\hline \multirow[t]{2}{*}{ Subgroup } & \multirow[t]{2}{*}{ Genetic model } & \multirow{2}{*}{$\frac{\text { Sample size }}{\text { Case/Control }}$} & \multirow[t]{2}{*}{ Type of model } & \multicolumn{2}{|c|}{ Test of heterogeneity } & \multicolumn{2}{|c|}{ Test of association } & \multicolumn{2}{|c|}{ Test of publication bias } \\
\hline & & & & $P^{2}$ & $P$ & OR & $95 \% \mathrm{CI}$ & $\mathrm{z}$ & $\mathrm{P}$ \\
\hline \multirow[t]{4}{*}{ Overall } & GlnGln $v s$ ArgArg & $2051 / 2919$ & Random & $59.1 \%$ & 0.01 & 1.29 & $0.90-1.85$ & 0.00 & 1.00 \\
\hline & GlnGln $v s$ ArgGln & & Fixed & $10.3 \%$ & 0.35 & 1.15 & $0.93-1.43$ & 0.00 & 1.00 \\
\hline & Dominant model & & Fixed & $46.1 \%$ & 0.05 & 0.80 & $0.66-0.99$ & 0.00 & 1.00 \\
\hline & Recessive model & & Random & $72.5 \%$ & 0.00 & 1.18 & $0.93-1.49$ & 0.00 & 1.00 \\
\hline \multirow[t]{4}{*}{ Asians } & GlnGln $v s$ ArgArg & $1759 / 2497$ & Random & $0.0 \%$ & 0.44 & 1.03 & $0.80-1.33$ & 0.00 & 1.00 \\
\hline & GlnGln vs ArgGln & & Fixed & $13.6 \%$ & 0.33 & 1.03 & $0.78-1.35$ & 0.00 & 1.00 \\
\hline & Dominant model & & Random & $51.9 \%$ & 0.04 & 0.87 & $0.59-1.26$ & 0.00 & 1.00 \\
\hline & Recessive model & & Random & $55.9 \%$ & 0.02 & 1.25 & $1.02-1.53$ & 0.00 & 1.00 \\
\hline \multirow[t]{4}{*}{ Caucasians } & GlnGln $v s$ ArgArg & $292 / 422$ & Random & $84.0 \%$ & 0.01 & 1.30 & $0.40-4.26$ & 0.00 & 1.00 \\
\hline & GlnGln $v s$ ArgGln & & Fixed & $0.0 \%$ & 0.59 & 1.58 & $1.03-2.43$ & 0.00 & 1.00 \\
\hline & Dominant model & & Fixed & $0.0 \%$ & 0.33 & 0.66 & $0.44-0.99$ & 0.00 & 1.00 \\
\hline & Recessive model & & Random & $93.6 \%$ & 0.00 & 0.88 & $0.22-3.39$ & 0.00 & 1.00 \\
\hline
\end{tabular}

\section{Publication bias}

Begg's funnel plot and the Egger test were used to assess publication bias. There was no evidence of publication bias in our study (Figure 3). The results suggested that there was no publication bias in this meta-analysis (all $\mathrm{P}>0.05$ ).

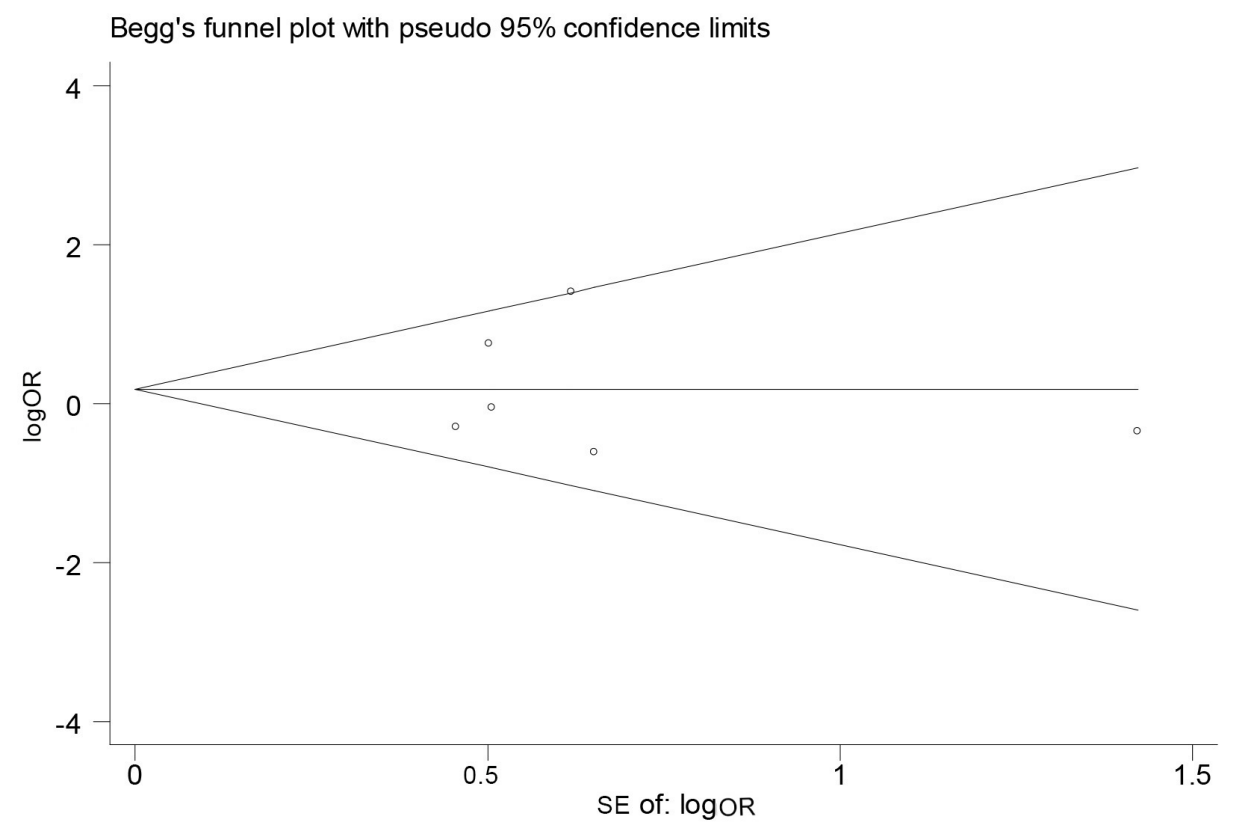

Figure 3. Begg funnel plot test of publication bias for the association of the XRCC1 Arg399Gln polymorphism and cervical carcinoma in the dominant model.

\section{DISCUSSION}

Cervical carcinoma is the second most common malignancy among women worldwide, and continues to be a leading cause of cancer death in women. To reduce new cases of 
cervical carcinoma, risks for this cancer should be identified and controlled. It is well known that HPV infection is the main risk factor for the pathogenesis of cervical carcinoma. In addition, many reports have focused on investigating genes underlying the development and progression of cervical carcinoma, and the pathogenesis of cervical carcinoma may be influenced by multiple genetic factors. The XRCC1 protein is a key molecule in the base excision repair pathway in the DNA repair process, which plays a key role in the integrity and stability of the genome and the pathogenesis and progression of human cancers (Poehlmann et al., 2010). Recently, a variety of studies have focused on the association between XRCC1 Arg399Gln polymorphism and cervical carcinoma, but these studies reported conflicting results. The most likely reason for the inconsistencies between these studies is that they are single case-control studies with small sample sizes. To address these issues, we carried out a systematic review and meta-analysis of all eligible case-control studies to estimate the risk of cervical carcinoma associated with the XRCC1 Arg399Gln polymorphism.

In the present meta-analysis, we searched as many publications as we could. The present meta-analysis, including 2051 cases and 2919 controls from 10 case-control studies, explored the association between the Arg399Gln polymorphism of the XRCC1 gene and cervical carcinoma risk. To the best of our knowledge, this is the first meta-analysis of the comprehensive assessment of the relationship between XRCC1 Arg399Gln polymorphism and the risk of cervical carcinoma. Overall, we found that there was significant association between this polymorphism and cervical carcinoma risk in the overall population. Because of the difference in genetic backgrounds and the environment in which the patients lived, we performed an ethnicity-specific subgroup analysis, and we also found a significant association between XRCC1 gene Arg399Gln polymorphism and cervical carcinoma risk in both Caucasians and Asians. Further sensitivity analysis confirmed the significant association between maternal Arg399Gln polymorphism and cervical carcinoma risk. No evidence showed publication bias in this meta-analysis for Arg399Gln polymorphism. As the eligible study number was limited in the meta-analysis, these results still need further investigation.

The potential effect of XRCC1 gene Arg399Gln polymorphism might be affected via gene-gene and gene-environment interactions. Evidence suggests that the linkage disequilibrium of Arg 194Trp and Arg399Gln may synergistically increase the risk of cervical carcinoma (Huang et al., 2007). In addition, significantly elevated risk of cervical cancer has been associated with the XRCC1 399GlnGln genotype among nonsmokers but not smokers (Niwa et al., 2005). Further studies of gene-gene and gene-environment interactions should be taken into consideration in future analysis, which should lead to a better comprehensive understanding of the association.

Some limitations of our meta-analysis should be mentioned. First, our meta-analysis was based on unadjusted OR estimates because not all published studies presented adjusted ORs, or when they did, the ORs were not adjusted by the same potential confounders, such as age, gender and exposures. The lack of information for the data analysis could cause serious confounding bias. Second, lack of original data limited our further evaluation of potential gene-gene and gene-environment interactions. Third, the number of studies and the number of subjects in the studies included in the meta-analysis by specific subgroups were small.

In conclusion, this meta-analysis suggests that the XRCC1 Arg399Gln polymorphism may be associated with cervical carcinoma risk. Further studies estimating the effect of gene-gene and gene-environment interactions may eventually provide a better comprehensive understanding of the association between XRCC1 Arg399Gln polymorphism and cervical carcinoma risk. 


\section{REFERENCES}

Barbisan G, Pérez LO, Difranza L, Fernández CJ, et al. (2011). XRCC1 Arg399Gln polymorphism and risk for cervical cancer development in Argentine women. Eur. J. Gynaecol. Oncol. 32: 274-279.

Boyle P, Leon ME, Maisonneuve P and Autier P (2003). Cancer control in women. Update 2003. Int. J. Gynaecol. Obstet. 83: $179-202$.

Caldecott KW (2003). XRCC1 and DNA strand break repair. DNA Repair 2: 955-969.

Caldecott KW, Aoufouchi S, Johnson P and Shall S (1996). XRCC1 polypeptide interacts with DNA polymerase beta and possibly poly (ADP-ribose) polymerase, and DNA ligase III is a novel molecular nick-sensor in vitro. Nucleic Acids Res. 24: 4387-4397.

Crosbie EJ, Einstein MH, Franceschi S and Kitchener HC (2013). Human papillomavirus and cervical cancer. Lancet 382: 889-899.

Huang J, Ye F, Chen H, Lu W, et al. (2007). The nonsynonymous single nucleotide polymorphisms of DNA repair gene $\mathrm{XRCC} 1$ and susceptibility to the development of cervical carcinoma and high-risk human papillomavirus infection. Int. J. Gynecol. Cancer 17: 668-675.

Jiang W, Wang ML, Zhang ZZ, Chen XJ, et al. (2009). The relationship between XRCC1 polymorphisms and the risk of cervical cancer in Jiangsu population. Acta Univ. Med. Nanjing 29:1-6.

Kim SU, Park SK, Yoo KY, Yoon KS, et al. (2002). XRCC1 genetic polymorphism and breast cancer risk. Pharmacogenetics 12: $335-338$.

Lee SG, Kim B, Choi J, Kim C, et al. (2012). Genetic polymorphisms of XRCC1 and risk of gastric cancer. Cancer Lett. 187: 53-60.

Ma WP, Jin P and Guo Y (2011). Single nucleotide polymorphisms of the DNA repair genes XPD and XRCC1 and the susceptibility to cervical squamous cell carcinoma. Prog. Obstet. Gynecol. 20: 881-885.

Niwa Y, Matsuo K, Ito H, Hirose K, et al. (2005). Association of XRCC1 Arg399Gln and OGG1 Ser326Cys polymorphisms with the risk of cervical cancer in Japanese subjects. Gynecol. Oncol. 99: 43-49.

Park JY, Lee SY, Jeon HS, Bae NC, et al. (2002). Polymorphism of the DNA repair gene XRCC1 and risk of primary lung cancer. Cancer Epidemiol. Biomarkers Prev. 11: 23-27.

Poehlmann A and Roessner A (2010). Importance of DNA damage checkpoints in the pathogenesis of human cancers. Pathol. Res. Prac. 206: 591-601.

Roszak A, Lianeri M and Jagodzinski PP (2011). Involvement of the XRCC1 Arg399Gln gene polymorphism in the development of cervical carcinoma. Int. J. Biol. Markers 26: 216-220.

Suh DH, Kim JW, Aziz MF, Devi UK, et al. (2010). Asian society of gynecologic oncology workshop 2010. J. Gynecol. Oncol. 21: 137-150.

Wannapa Settheetham-Ishida, Yuenyao P, Natphopsuk S, Settheetham D, et al. (2011). Genetic risk of DNA repair gene polymorphisms (XRCC1 and XRCC3) for high risk human papillomavirus negative cervical cancer in Northeast Thailand. Asian Pac. J. Cancer Prev. 12: 963-966.

Wu MT, Liu CL and Wu TN (2004). Genetic polymorphism of p53 and XRCC1 in cervical intraepithelial neoplasm in Taiwanese women. J. Formos Med. Assoc. 103: 337-343.

Xiao HY, Wu WQ, Xie HY and Bao XM (2010). Relationship between the polymorphism of XRCC1-Arg399Gln and incidence risk of cervical cancer in the population of Guangdong. Hainan Med. J. 21: 35-37.

Yang HP, Gonzalez Bosquet J, Li Q, Platz EA, et al. (2010). Common genetic variation in the sex hormone metabolic pathway and endometrial cancer risk: pathway-based evaluation of candidate genes. Carcinogenesis 31: 827-833.

Zhang L, Ruan Z, Hong Q, Gong X, et al. (2012). Single nucleotide polymorphisms in DNA repair genes and risk of cervical cancer: A case-control study. Oncol. Lett. 3: 351-362.

Zucchetto A, Serraino D, Polesel J, Negri E, et al. (2009). Hormone-related factors and gynecological conditions in relation to endometrial cancer risk. Eur. J. Cancer Prev. 18: 316-321. 